\title{
TTR
}

Traduction, terminologie, re?daction

\section{" Die sache selbs, der sprachen art, ein christlich hertz " - Les principes d'une théorie de la traduction selon Martin Luther}

\section{Hans-Herbert S. Räkel}

Volume 3, numéro 2, 2e semestre 1990

La traduction des textes sacrés : le domaine biblique

URI : https://id.erudit.org/iderudit/037070ar

DOI : https://doi.org/10.7202/037070ar

Aller au sommaire du numéro

Éditeur(s)

Association canadienne de traductologie

ISSN

0835-8443 (imprimé)

1708-2188 (numérique)

Découvrir la revue

Citer cet article

Räkel, H.-H. S. (1990). « Die sache selbs, der sprachen art, ein christlich hertz "

- Les principes d'une théorie de la traduction selon Martin Luther. TTR, 3(2),

81-95. https://doi.org/10.7202/037070ar

Tous droits réservés @ TTR: traduction, terminologie, rédaction — Les auteurs, 1990
Cecument est protégé par la loi sur le droit d'auteur. L’utilisation des services d'Érudit (y compris la reproduction) est assujettie à sa politique d'utilisation que vous pouvez consulter en ligne.

https://apropos.erudit.org/fr/usagers/politique-dutilisation/ 


\section{«Die sache selbs, der sprachen art, ein christlich hertz» ${ }^{1}$ - Les principes d'une théorie de la traduction selon Martin Luther}

\section{Hans-Herbert S. Räkel}

\section{L'emblème}

«Par économie, je ne prononcerai que le nom propre de Luther, l'emblème en suffira»: partons de cette remarque de Jacques Derrida $(1985$, p. 26), faite dans l'introduction d'une conférence dont le titre fut "Théologie de la traduction». Derrida y souligne un aspect fondamental de la philosophie de Schelling selon laquelle l'homme est appelé à «développer (entwickeln) ce qui fait défaut dans la manifestation totale de Dieu». Derrida affirme que «c'est ce qu'on appelle la traduction» (p. 39). Cette conclusion est l'aboutissement logique et nécessaire en quelque sorte de cette «configuration prémoderne» du "romantisme allemand qui fut a la fois un moment de réflexion intense, agitée, tourmentée, fascinée sur la traduction, sa possibilité, sa nécessité, sa signification pour la langue et la littérature allemandes $e t$ un moment où une certaine pensée de la Bildung, de l'Einbildung et de toutes les modifications du Bilden ne se sépare pas de ce qu'on pourrait appeler justement l'impératif de la traduction, la tâche du traducteur, le devoir-traduire» (pp. 26-27). Luther reste, pour Derrida, en-deça de cette configuration. L'emblème de son nom concrétise plutôt le fait que «des langues naturelles se sont fixées [...] à l'événement même de la

1. «La chose elle-même, la nature de la langue, un cœur chrétien.» 
traduction de la Bible» (p. 26). Sans aucun doute, le nom de Luther évoque cette naissance de la langue allemande par la traduction et une sorte de paternité du réformateur. Mais ce nom évoque aussi d'autres aspects, celui d'une traduction respectant entièrement la langue-cible, celui du retour aux sources originales, celui d'un texte devenu en quelque sorte canonique malgré les intentions du traducteur. En étudiant l'impact de la traduction sur la langue allemande au moment de sa conversion au christianisme à l'époque carolingienne $^{2}$, j'ai ressenti le besoin de réexaminer les principes qui ont guidé le traducteur Luther, d'en faire un relevé plus complet et peut-être plus véridique que l'idée qu'évoque l'emblème de son nom.

L'intense réflexion qu'a suscitée ces demières années la recherche en théorie de la traduction nous a imposé de nouvelles perspectives, de sorte que les commentaires des traducteurs, qui avaient eu un statut de source auxiliaire, ont acquis la valeur de sources primaires et originales pour scruter l'activité traduisante en soi plutôt que ses résultats.

\section{L'œuvre personnelle}

Luther a écrit deux textes théoriques sur sa propre activité de traducteur: Ein Sendbrieff D. M. Luthers. Von Dolmetzschen vnd fürbitt der Heiligen. M.D.XXX $X^{3}$ (Luther, 1530), que j'appellerai la Lettre, écrite en 1530 à la suite de la révision du Nouveau Testament, et Summarien vber die Psalmen, vnd Vrsachen des Dolmetschens $^{4}$, que j'appellerai les Principes, parus en 1533 à la suite d'une révision de la traduction des Psaumes (Luther, 1533).

2. Hans-Herbert S. Rukel, «les Barbarismes d'Otfrid de Wissembourg: traduction et exégèse vernaculaires en vieux-haut allemand», TTR, Vol. II, $\mathrm{n}^{\circ} 1$ (1989), pp. 89-102.

3. Le titre original et la première partie du texte (sans le passage important concernant «die sache selbs») se trouve à l'annexe de la Bible (Luther, 1545 , pp. $242^{*}-249^{\circ}$ ). La version que présente Hans Joachim Störig (1969) a l'avantage et l'inconvénient d'une version modemisée archaïsante. Je cite l'édition de Clemen (Luther, 1530).

4. Luther, 1545 (Annexe Anhang und Dokumente), pp. $250^{\circ}$ à $257^{\circ}$. 
Ces deux écrits sont polémiques, le premier plus que le second, et en les relisant plusieurs siècles après leur parution on a peut-être tout simplement l'impression que Luther a raison dans tout ce qu'il défend. Pour nous, il ne s'agira pas de rouvrir ce dossier. Nous chercherons plutôt les motifs et les convictions profondes qui sous-tendent son argumentation. Pour ce faire un témoignage partisan comme la préface dont son ami Wenzeslaus Link a fait accompagner le texte de la Lettre est aussi révélateur que les propos du réformateur lui-même. Après avoir mentionné que les ennemis de la vérité prétendent que la traduction du Nouveau Testament aurait changé ou faussé le sens du texte à beaucoup d'endroits («geendert / odder auch verfelschet)), il exprime l'espoir que la Lettre fera taire les détracteurs et rassurera les fidèles. Puis il ajoute ceci: «Peut-être ce sera un encouragement pour qu'on écrive un peu plus sur cette matière. $\rangle^{5} \mathrm{Ce}$ que je voudrais retenir de ce souhait, c'est l'idée que l'on puisse et que l'on doive discuter, voire commenter la nouvelle traduction de la Bible. Cette position semble également être celle de Luther luimême. Il considère sa traduction comme une œuvre personnelle: "C'est mon testament et ma traduction, il est et restera le mien», et un peu plus tôt: «[...]ce faisant je n'ai obligé personne à le lire et ne l'ai fait que pour être utile à ceux qui ne peuvent faire mieux. Il n'est point interdit d'en faire un meilleur. ${ }^{6}$ En tant qu'œuvre personnelle, la traduction est la propriété du traducteur, ce qui

5. Le texte de Luther est cité selon ma propre traduction. "Villeicht auch verursachet / das ettwas mehrers auff solche fragstuck odder materi / geschriben werde» (Luther, 1530, p. 179, 18-19).

6. «Es ist mein testament vnd mein dolmetschung / vnd sol mein bleiben vnnd sein» (Luther, 1530, p. 180, 33-34); «habe damit niemand gezwungen / das ers lese / sondern frey gelasen / und allein zu dienst gethan denen / die es nicht besser machen können / Ist niemandt verboten ein bessers zu machen» (29-31). Luther reprend cette idée plus loin: «Wer mein dolmetzschen nicht wil / der las es anstehen" (p. 186, 34). Dans les Principes, il dit également que sa traduction est faite pour ses fidèles et ceux qui l'apprécient: «Wer vnser Dolmetschen nicht haben wil / der lasses / Wir dienen damit den vnsern / vnd die es gerne haben.» (Luther, 1533, p. 257", 38-39) 
interdit le plagiat ${ }^{7}$ aussi bien que toute ingérence dans le texte. Bien sûr, le traducteur en assume aussi l'entière responsabilité. Dans les Principes, une remarque insiste sur cet aspect: «[...] qui voudrait avoir la prétention d'avoir évité, comme s'il était lui-même le Christ et le Saint-Esprit, la moindre faute? $»^{8}$

\section{Le travail d'équipe}

Cette responsabilité est un lourd fardeau et l'on comprend que Luther ait cherché à minimiser les sources d'erreur en consultant d'abord ses collègues et amis, ensuite les spécialistes éminents de toutes les disciplines liées à la traduction de la Bible. ${ }^{9}$ Il est alors très significatif que sa principale critique de la traduction de saint Jérôme, qu'il considère par ailleurs comme prédécesseur et modèle, touche le fait qu'il a travaillé seul: «S. Hieronymus hat fur ein person gnug gethan. Nulla enim privata persona tantum efficere potuisset. Si unum atque alterum coniunxisset sibi, Spiritus Sanctus affuisset illis iuxta illud: 'Ubi duo vel tres' etc. Nec translatores debent esse soli, denn eim einigen fallen nicht allzeit gut et propria verba ${\mathrm{zu} .{ }^{10}}^{10} \mathrm{~J}$ 'insiste ici sur le lien que Luther établit entre les avantages techniques du travail en équipe et le mystère ecclésiastique dont parle Jésus lorsqu'il fait la promesse citée par saint Mathieu $(18,20)$ : "Que deux ou trois soient réunis en mon Nom, je

7. «Wie wol / was das fur ein tugent sey / einem andern sein buch lestern vnd schenden / darnach dasselbige stelen / vnd vnter eigenem namen dennoch auß lassen gehen [...]» (Luther, 1530, p. $182,8-10)$.

8. «Denn wer wil so vermessen sein / das er / gleich als were er Christus vnd der heilige geist selbs / kein wort / wolt gefeilet haben?» (Luther, 1533, p. 257", 11-12)

9. Dans ses préfaces, il en rend compte, par exemple pour la traduction du Livre de la Sagesse : enemlich das buch von der Weisheit / mit hülff meiner guten freunde verdeudscht / und so viel vns Got verlihen / aus dem finstern latinschen vnd Griechischen / ynn das deudsche liecht gebracht» (cf. note 13; Luther, 1545, p. $240^{*}, 8-10$ ).

10. Propos de table (Weimarer Ausgabe Tischreden Band 1, p. 486, 19-23) cité d'après Luther (1545, p. 238*, note 2). 
suis là au milieu d'eux.» Il ne doute évidemment pas de ce que cette promesse est réelle et s'applique à plus forte raison à son équipe de traducteurs. Ce n'est donc point une répétition de la légende séculaire qui raconte comment les Septante, par miracle, ont écrit tous le même texte en traduisant la Bible de l'hébreu en grec. Ce n'est pas non plus l'affirmation pure et simple d'un principe d'efficacité par le travail d'équipe, c'est une synthèse dialectique de ces deux principes, qui respecte la science et l'effort humain, mais qui maintient qu'aucun effort humain n'aboutira sans la grâce. Ainsi, le traducteur peut concevoir l'idée du progrès et inviter son lecteur à participer à l'aventure de la traduction. La postface du psautier allemand de 1531 ne laisse aucun doute à ce sujet: d'abord, le traducteur affirme que beaucoup d'érudits ont participé à la tâche (il est vrai qu'il avait réuni dans sa maison, plusieurs fois par semaine, pendant deux mois à partir du mois de janvier 1531, tous les spécialistes concernés de près ou de loin par les psaumes, leur langue, leur histoire, leurs traductions). Puis il ajoute: «Néanmoins nous conservons également le texte de notre édition précédente du psautier, pour ceux qui voudraient examiner notre démarche et suivre nos traces pour observer comment la traduction peut s'approcher de plus en plus de l'usage. Car le psautier précédent est souvent plus près de l'hébreu et plus loin de l'allemand, tandis que celui-ci est plus près de l'allemand et plus loin de l'hébreu.»"

S'approcher de plus en plus de la langue-cible sans sacrifier le texte, voilà une idée qui confirme ce que nous avons remarqué auparavant: la traduction ne saurait être définitive et, par conséquent, ne saurait être canonique non plus, même si, dans certaines circonstances, elle peut paraître achevée, comme Luther le pensait de sa propre traduction des Psaumes. ${ }^{12}$

11. «Doch lassen wir unsern vorigen deutschen Psalter (1524/28) auch bleiben um der willen, so da begehren, zu sehen unser Exempel und Fußstapfen, wie man mit Dolmetschen năher und näher kommt; denn der vorige deutsche Psalter ist an viel Orten dem Ebräischen năher und dem Deutschen ferner, dieser ist dem Deutschen näher und dem Ebräischen femer.» (Luther, 1545, p. 91*)

12. «[...]dabei wirs gedenken hinfort bleiben zu lassen.» (Ibid.) 
Malgré le caractère nécessairement inachevé, cette traduction peut prétendre à un statut supérieur, d'abord aux psaumes latins qui ne sont qu'une autre traduction manifestement défectueuse, mais supérieur aussi au texte original écrit dans une langue morte: traduire les psaumes dans une langue vivante, c'est en quelque sorte les ressusciter, ouvrir une voie à la parole de Dieu, la sortir des ténèbres pour l'exposer au jour. ${ }^{13}$

Voilà pourquoi le respect de la langue-cible chez Luther est autre chose qu'une technicalité, autre chose qu'un symptôme de l'affirmation des langues vernaculaires au $16^{\circ}$ siècle.

\section{La nature de la langue}

Le respect de la langue-cible, voilà le premier principe du traducteur Luther, principe d'ailleurs bien connu, mais non exempt d'un fondement théologique essentiel. Ce premier principe correspond en somme parfaitement à ce que Derrida a appelé l'emblème du nom de Luther.

Luther lui-même parle à ce sujet du respect de la «nature de la langue ${ }^{14}$ Toute la première partie de la Lettre est consacrée à démontrer que l'adverbe 'allein', qu'il avait ajouté au texte original de l'épître aux Romains ${ }^{15}$ s'y trouve bien à sa place en raison des règles de la langue allemande. Or, on constate que Luther a un peu forcé son argumentation, puisque l'adverbe 'allein' qui renforce le contraste entre un élément nié et un élément affirmé appartient

13. Luther dit par exemple qu'il a sorti le Livre de la Sagesse des ténèbres latines et grecques pour l'exposer à la clarté allemande (cf. note 9; «aus dem finstem latinschen vnd Griechischen / ynn das deudsche liecht gebrachts, Luther, 1545, p. $240^{\circ}$ ). Le texte original du Livre de la Sagesse est en effet écrit en grec.

14. «die art vnser deutschen sprache» (Luther, 1530, p. 184, 9); «...vnd ist yhr art (Ibid., 184, 17); «art der sprachen» (Ibid., 187, 35); «der sprachen art» (Ibid., 187, 36); «dz zwinget die sache selbs neben der sprachen art (Ibid., 188, 15-16). La forme 'sprachen' peut représenter un génitif du pluriel ou un génitif du singulier, cf. note 19).

15. 3,28: «ex fide absque operibus». 
tout au plus au domaine de la norme sinon à celui du style. Cette norme ou cette préférence stylistique n'est d'ailleurs pas exclusivement allemande, puisque la phrase latine tolère parfaitement l'adverbe 'solum' ou 'tantum' dans la même position, même si à l'endroit en question saint Jérôme ne l'a pas utilisé.

Mais la nature de la langue ne se limite pas à la grammaire, elle marque aussi l'usage établi du discours ${ }^{16}$ et la tradition des métaphores ${ }^{17}$. Je n'insiste pas plus longtemps sur ce principe bien connu que le réformateur a lui-même résumé à plusieurs reprises. $^{18}$

\section{La force des choses}

Si Luther attache une si grande importance à ce qu'il appelle «der sprachen arts, il ne le fait pourtant pas pour des raisons linguistiques. La meilleure preuve en est la Lettre elle-même, dont la

16. Ainsi, dans les Principes, Luther se justifie d'avoir remplacé le pronom 'mea' par 'tua' au verset 9 du psaume 91, parce que le contexte parle à une deuxième personne et parce qu' «une personne de langue allemande ne peut pas comprendre le brusque changement de personne» ("Vnd der gemein Deudsch man die plötzliche verenderung der personen im reden nicht wol kan mercken», Luther, 1533 , p. $\left.255^{*}, 18-19\right)$.

17. Plusieurs fois il mentionne avoir changé l'image où l'hébreu utilise l'idée de 'gras' (fett), terme qu'il remplacera par quelque chose de moins métaphorique et plus habituel, comme 'fruchtbar' (fertile) et 'frisch' (frais).

18. «[...] den man mus nicht die buchstaben inn der lateinischen sprachen fragen / wie man sol Deutsch reden» (Luther, 1530, p. 184,23 ). "Vnd so mans solte allenthalben von wort zu wort / vnd nicht das mehr mal nach dem synn verdolmestchen (wie die iuden vnd vnuerstendige dolmetscher wöllen) wurde es niemant verstehen mugen» (Luther, 1545, p. 239*, 25-27). «[...] das wir so frey / an vielen orten / von den buchstaben gangen sind" (Luther, 1533 , p. $\left.250^{*}, 8-9\right)$. «Denn wir die regel gehalten / Wo die wort haben mügen leiden vnd geben / einen bessern verstand / $\mathrm{Da}$ haben wir vns nicht lassen zwingen... Wie denn alle Schulmeister leren /das nicht der sinn den worten / sondern die wort dem sinn dienen vnd folgen sollen.» (Luther, 1533, p. 252*, 21-26) 
deuxième partie transcende délibérément ce stade de la réflexion: «Or, je ne me suis point référé exclusivement à la nature de la langue.. ${ }^{19}$ Le texte de saint Paul expose ici des faits précis, la base même du christianisme. Luther se sent le droit d'aller au-delà de la simple équivalence fonctionnelle en insistant sur le fond de la question qui est théologique: "C'est la force des choses en plus de la nature de la langue» qui motive la décision du traducteur d'ajouter l'adverbe «allein» à sa version traduite de l'épître aux Romains. $\rangle^{20} \mathrm{Ce}$ n'est donc pas une question de code, c'est une question de contenu du message. Voilà que la Lettre devient elle-même «kerygma», prédication plutôt que discussion. Il est clair maintenant que la traduction elle aussi poursuit ce même but: elle n'est point la parole de Dieu, mais elle peut le devenir à chaque lecture si Dieu le veut. Peu importe le texte original, c'est-à-dire le niveau du signifié rattaché à ses signifiants, pourvu que l'interprétation («meinung») puisse se faire.

19. «Aber nu hab ich nicht allein der sprachen art vertrawet vnd gefolget» (Luther, 1530, p.187, 35-36). 'Der sprachen art' peut être un génitif du pluriel, mais également un génitif du singulier de la déclinaison faible, cf. «der Ebreischen sprachen raum lassen» (cf. note 22) et «inn der lateinischen sprachen» (cf. note 18): datif du singulier! Dans un contexte différent, Luther utilise toutefois le génitif de la déclinaison forte: «die art vnser deutschen sprache». (Luther, 1530, p. 184, 9)

20. «dz zwinget die sache selbs neben der sprachen art» (Luther, 1530 , p. 188, 15-16). Luther se réfere à cette notion de «chose» à d'autres endroits moins pertinents pour les fondements de la foi, mais néanmoins importants en tant que message: au verset 12 du psaume 118 il fait appel au contexte, mais aussi à la «chose», voire à la force des choses, qui lui permet de prendre une autre décision de critique textuelle que celle que la tradition juive avait adoptée. Est-ce que les impies seront brûlés comme les ronces sous le chaudron avant même que la chaleur fasse son effet, ou est-ce que les impies accourent de toutes parts pour étouffer l'esprit qui anime les fidèles? Contexte et sens, «die sache selbs», exigent une décision sans appel, et Luther se moque de l'interprétation juive, où les impies semblent devoir être abattus après être tombés en cendres... (Luther, 1533, pp. 256*, $40-257^{*}, 3$ ) 


\section{6. À la lettre}

Or, il y a un aspect chez Luther qui semble contredire la logique de la position décrite jusqu'alors.

Nous le trouvons très clairement exprimé dans un bref passage de la Lettre, entre la première partie qui parle de la nature de la langue et la deuxième partie qui parle de la force des choses: «Je n'ai toutefois pas pris trop de liberté en ce qui concerne les lettres, au contraire, moi-même et mes acolytes, nous avons mis beaucoup de soin, aux endroits où cela importe, de respecter le texte à la lettre. $\rangle^{21}$

Cette position n'est pas sans nous intriguer: le réformateur ne se réserve-t-il pas tout simplement le droit d'en faire à sa tête? Pourquoi à tel endroit trouve-t-il important de conserver une formule qui ne respecte pas la nature de la langue et manque même de clarté, tandis qu'ailleurs, il fait des efforts démesurés pour défendre par exemple son adverbe 'allein'? Il y a différentes raisons à cette contradiction apparente dans l'attitude du réformateur. Une première est fournie par le respect de la nature de l'hébreu. En effet, on a l'impression que le réformateur part de l'idée fondamentale qui veut que tout puisse être exprimé adéquatement dans sa langue allemande, pourvu que l'on fasse l'effort de s'en approcher de plus en plus. Or, il y a des passages où il constate que l'hébreu exprime simplement mieux l'idée en question que l'allemand. Dans ce cas, il faut conserver la formule hébräque et éduquer l'allemand à l'imiter. Ainsi, Luther a conservé la formule du psaume 68,19: «Du hast das Gefängnis gefangen» (tu as emprisonné la prison) où, d'après lui, «tu as libéré les prisonniers» aurait

21. «Doch hab ich widerumb nicht allzu frey die buchstaben lassen faren / Sondern mit grossen sorgen sampt meinen gehülffen drauff gesehen / das wo etwa an einem ort gelegenn ist / hab ichs nach den buchstaben behalten.» (Luther, 1530, p. 187, 2022) La même chose est répétée dans les Principes : «Parfois, au contraire, nous avons traduit carrément mot à mot bien que nous eussions pu donner une version différente plus claire. La raison en est que ces mots sont importants." - «Widderumb haben wir zu weilen / auch stracks den worten nach gedolmetscht / ob wirs wol hetten anders vnd deudlicher künnen geben / Darumb / das an den selben worten etwas gelegen ist.» (Luther, 1533, p. 254, 3-5; cf. aussi p. $257^{*}, 26-28$ ) 
été meilleur en allemand. Mais, étant donné que c'est une formule stylistique fréquente et qu'elle insiste de façon tout à fait particulière sur la délivrance, on doit «pour l'honneur de la doctrine et le réconfort de notre conscience conserver ces mots, les rendre habituels et céder la place à la langue hébraïque dans les cas où elle réussit mieux que l'allemand ne pourrait le faire». ${ }^{22}$

Mais il y a bien sûr des cas beaucoup plus complexes: en traduisant l'Ancien Testament, notamment les Psaumes, Luther s'est trouvé confronté à la tradition d'interprétation rabbinique. Le rapport qu'il établit avec cette tradition a quelque chose de particulier dans la mesure où il distingue de façon très claire entre deux niveaux de signification dont le premier pourrait être appelé «niveau du signifié» dans le sens que de Saussure donne à ce terme, et le deuxième «niveau de l'interprétation». Luther dit «meinung», ce qui inclut le message religieux. Or, il est curieux de voir qu'en comparant l'exégèse rabbinique et la sienne, il ne s'arrête pas au niveau du signifié. Le verset 10 du psaume 58 par exemple parle des impies et les compare selon la tradition rabbinique aux ronces qui brûlent sous un chaudron, mais qui sont consumées avant de le chauffer. Luther écrit: «Nous acceptons cette interprétation et c'est aussi la nôtre, mais nous l'avons rendue ainsi: Avant que les ronces ne mûrissent, voire ne prennent de l'envergure dans le maquis, la colère, c'est-à-dire une hache, tombera sur elles tant qu'elles sont encore vertes et tendres. ${ }^{23}$ L'interprétation semble en effet la même: les impies n'ont aucune chance d'exécuter leurs mauvaises intentions; mais au niveau des signifiés les deux versions sont tout à fait incompatibles. Dans un cas comme celui-ci, Luther ne critique pas la tradition rabbinique et admet que l'on puisse atteindre le but de l'interprétation («meinung») par des voies totalement différentes. C'est pourquoi il choisira tantôt une formule plus allemande, tantôt une formule plus hébraïque, tantôt sa propre solution. Et s'il ne germanise pas trop les passages obscurs, c'est aussi parce qu'il a, à l'occasion, une troisième raison: il croit à une herméneutique très moderne: «Nous avons voulu laisser la liberté à chacun de

22. «Darumb müssen wir zu ehren solcher lere / vnd zu trost vnsers gewissens / solche wort behalten / gewonen vnd also der Ebreischen sprachen raum lassen / wo sie es besser macht / denn vnser Deudsche thun kann.» (Luther, 1533, p. 254", 18-20)

23. «Diese meinung lassen wir gut sein / vnd ist auch die vnsere.» (Luther, 1533, p. 250*, $23-251^{*}, 1$ ) 
comprendre selon les dispositions et les dimensions de son esprit; sinon, nous aurions choisi une traduction qui ne donnerait que notre propre compréhension. ${ }^{24}$ Ceci permet donc aussi l'acceptation d'interprétations divergentes. Luther donne l'exemple d'un passage qu'il a traduit dans le sens de sa propre interpétation, mais il ajoute: «Je sais parfaitement que saint Bernard l'a compris autrement et je le lui accorde [...] Que d'autres l'interprètent encore autrement, nous leur laissons la conviction de leur interprétation. Notre interprétation est également bonne, si elle n'est peut-être pas la meilleure. ${ }^{25}$

\section{Le cœur}

Comment le réformateur peut-il ainsi laisser libre cours à toutes les inspirations, accorder à des prédécesseurs comme à ses contemporains et même aux Juifs le bénéfice d'interprétations multiples? Il dispose malgré tout d'un critère, unique et essentiel, c'est son propre concept de la foi et la christologie qui y est rattachée. Pour Luther, l'Ancien Testament n'a qu'une seule fonction, c'est de préparer la venue du Christ. En ceci il est fidele à la tradition des tout premiers chrétiens. Puisque cet aspect de l'Ancien Testament n'entre pas en ligne de compte pour les Juifs, il est clair qu'ils ne peuvent pas non plus entendre ou faire entendre le message qui y est contenu. Luther le dit et le redit, par exemple dans la préface de l'Ancien Testament de 1523: «Je pense que si la Bible doit être publiée, c'est nous les chrétiens qui devons nous en charger, puisque nous avons la connaissance du Christ, sans laquelle la connaissance de la langue n'est d'aucun secours. ${ }^{26}$ Il peut citer

24. «Darumb haben wir einem jglichen wollen raum lassen / nach seines geists gaben vnd masse / die selbigen zu verstehen / Sonst hetten wir sie wol also verdeudscht / damit vnser verstand hette erkand mügen werden.» (Luther, 1533, p. 254*, 26-28)

25. Ibid., p. $255^{*}, 8-10$.

26. «Vnd achte / sol die Bibel erfur komen / so mussen wyrs thun / die Christen sind / als die den verstand Christi haben / on wilchen auch die kunst der sprache nichts ist.» (Luther, 1545, p. 238*, 19-21) Dans les Principes, il rappelle que le visage de Moïse est caché aux Juifs de sorte qu'ils saisissent mal et rarement le sens de l'Écriture, notamment dans les Prophètes: «So wissen wir auch / vnd S. Paulus 2.Cor.4 (3, 13 sq.) leret vns 
saint Paul pour appuyer sa position et il a hérité de toute la tradition médiévale d'interprétation allégorique pour la défendre. Ainsi il écrit dans la marge du psaume 68: «Ce psaume parle exclusivement du Christ. C'est pourquoi il faut y être très attentif, puisqu'il tient un discours étrange selon la lettre. $\aleph^{27}$ Ainsi il admet sans scrupule que les «rois» du psaume sont les apôtres et que la «maîtresse de la maison» est en réalité l'Église. ${ }^{28}$

Ici encore, Luther veut rester fidèle à une tradition spirituelle pour maintenir le dialogue avec le Dieu dont lui aussi croyait qu'il avait jadis choisi son peuple, tout en refusant la mystification systématique et facile de l'allégorie médiévale. Cette attitude, il l'a

/ das Moses angesicht den Juden verdeckt ist / das sie der schrifft meinung / sonderlich jnn den Propheten / wenig vnd selten treffen.» (Luther, 1545, p. 252*, 26-28) Plus loin dans le même texte il parlera d'une interprétation juive erronée du texte qui chante le Christ et son royaume: «Weil denn solchs ein Jüdisscher jrriger sinn ist im Text $/$ nnd wir wissen / das dieser Psalm von Christo vnd seinem Reich singet / Vnd die wort dieses vers / aus art der sprachen / diesen sinn geben... » (Luther, 1545, p. $256^{*}, 12-14$ )

27. «Dieser Psalm redet durchaus von Christo / Darumb mus man wol drauff mercken / Denn er furet seltzame rede vnd wort nach dem buchstaben.» (Luther, 1545, p. 1021)

28. Mais le réformateur a complètement changé d'avis au cours du quart de siècle qui a suivi sa découverte de la foi et sa conversion. En 1540, il dit à ses amis que dans sa jeunesse, avant d'aborder la théologie, il avait été un virtuose en allégories, tropologie, analogies; qu'il a abandonné toute cette magie pour comprendre que la traduction littérale est suffisante, qu'elle apporte vie, réconfort, force, savoir et connaissance, tandis que le reste n'est que charlatanerie, malgré son éclat: «Weil ich jung war, da war ich gelertt, vnd sonderlich, ehe ich in die theologia $\mathrm{kam}$, da gieng ich mitt allegoriis, tropologiis, analogiis vmb vnd machte lauter kunst; wens jtzt einer hette, er hilts vor eitell heiltumb. Ich weiß, das ein lauter dreck ist, den nuhn hab ichs faren lassen, vnd diB ist mein letzte vnd beste kunst: Tradere scripturam simplici sensu, denn literalis sensus, der thuts, da ist leben, trost, krafft, lehr vnd kunst inen. Das ander ist narren werck, wie wol es hoch gleist.» (Luthers Werke in Auswahl, éd. Otto Clemen, vol. 8 «Tischreden» - Propos de table. Berlin Walter de Gruyter, $3^{\circ}$ éd., 1962, p. 284, n' 5285). 
léguée à l'église luthérienne dans sa préface qui se veut justement un «guide pour chercher le Christ et l'évangile dans l'Ancien Testament. ${ }^{29}$ Ainsi, la foi est le critère, voire la condition nécessaire (mais non suffisante), pour que la parole de Dieu passe à travers un traducteur pour s'adresser à un lecteur. Si l'interprétation est un acte personnel, et Luther insiste pour l'affirmer, le traducteur doit avoir personnellement entendu la parole de Dieu, ni ses mots ni ses lettres, mais le message, la bonne nouvelle, l'évangile, pour pouvoir en donner une traduction méritant ce nom. En ce qui concerne l'Ancien Testament, cette remarque ne pose pas beaucoup de problèmes, les frontières entre la compréhension chrétienne et la compréhension juive sont connues et prévisibles. Mais le principe a des conséquences plus lourdes comme le prouve une petite parenthèse dans le texte de la Lettre qui semble avoir peu intéressé les commentateurs et dont le contenu est peut-être banal pour l'historien, mais qui, sur le fond de ce que nous venons d'esquisser, prend une certaine valeur: Luther affirme, dans une envolée assez solennelle, que la traduction exige «ein recht / frum / trew / vleissig / forchtsam / Christlich / geleret / erfarn / geübet hertz» - un cœur habitué, expérimenté, renseigné, chrétien, respectueux, diligent, fidele, pieux et droit. Mais Luther poursuit, et voilà que sa position montre toute la problématique insurmontable du cercle vicieux de la traduction: ne saurait traduire que celui qui comprend, ne saurait comprendre que celui qui a déjà traduit, ou mieux, à qui le savoir, c'est-à-dire la foi, a déjà été traduite: «C'est pourquoi j'estime qu'aucun faux chrétien, aucun esprit sectaire n'est capable de traduire fidèlement. ${ }^{30}$ Il sait de quoi il parle: la traduction des prophètes parue à Worms en 1527 , dont Luther reconnaît l'art et dans laquelle il remarque non sans respect que sa propre manière de traduire a fait des adeptes, a comme auteurs deux spiritualistes et anabaptistes, Ludwig Hătzer et Hans Denck, qui en plus ont fait appel à des juifs, lesquels n'ont naturellement pas montré de respect pour le Christ. La traduction de ces auteurs spiritualistes et juifs risque alors de rendre la parole de Dieu incompréhensible. Or, toute traduction de l'Écriture sainte ne saurait être autre chose qu'un service rendu à l'homme qui veut entendre la parole de Dieu. Entendre la parole de Dieu, c'est le salut.

29. «... anleitung / Christum vnd das Euangelium $z \mathfrak{u}$ suchen im alten Testament». (Luther, 1545, p. 20, 19-20)

30. «Darumb halt ich / das kein falscher Christ noch rottengeist trewlich dolmetzschen könne.» (Luther, 1530, p. 187, 28-30) 
Débarrassée de son déguisement dogmatique du seizième siècle, cette dernière conséquence d'une théorie de la traduction revient depuis sous la forme d'une pensée qui considère toute parole comme traduction. Derrida, le philosophe et historien, $n$ 'hésite pas, dans le sillage de Schelling, à évoquer la signification theologique d'une telle perspective. Dans cette perspective Martin Buber et Franz Rosenzweig ont entrepris une nouvelle fois de traduire la Bible en allemand. Je rendrai notamment hommage à ce dernier, dont l'étude Die Schrift und Luther ${ }^{31}$ a fixé solidement l'image du réformateur dans le panthéon de ceux qui ont participé à rendre audible le témoignage millénaire sinon éternel de la Bible.

Université de Montréal

\section{Références}

BERMAN, Antoine (1984). L'Épreuve de l'étranger: culture et traduction dans l'Allemagne romantique. Paris, Gallimard.

DERRIDA, Jacques (1985). «Langages et institutions de la philosophie». Texte, 4, pp. 9-39.

31. Störig, Hans-Joachim (éd. 1969), pp. 194-222. Cette étude avait été publiée séparément en 1926 ; elle fut réimprimée dans Martin Buber et Franz Rosenzweig, Die Schrift und ihre Verdeutschung, Berlin, Schocken, 1936. Antoine Berman (1984) a consacré le premier chapitre de son beau livre l'Épreuve de l'étranger à «Luther ou la traduction comme fondation». Je souscris pleinement à cette idée que Derrida a reprise et qui est également présente dans l'étude de Rosenzweig dont Berman traduit un long passage (pp. 50-51). Toutefois, j'ajouterai que Rosenzweig a su dégager de surcroît la configuration théologique très particulière de la traduction luthérienne («die Glaubensfrage des Obersetzens", p. 198), sur laquelle j'ai voulu attirer l'attention ici et qui postule que l'objet véritable de la traduction de la Bible ne soit pas le texte, mais le salut. 
LUTHER, 1530. «Sendbrief vom Dolmetschen. 1530». Luthers Werke in Auswahl, éd. Otto Clemen, vol. 4. Berlin, Walter

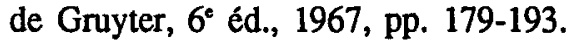

LUTHER, 1533. «Der erste Teil von 'Summarien vber die Psalmen, vnd Vrsachen des dolmetschens. Mart. Luther' (Wittenberg 1533)», in Luther, 1545, pp. 250*-257*.

LUTHER, 1545. D. Martin Luther, Die gantze Heilige Schrifft Deudsch. Wittenberg 1545, éd. Hans Volz, Heinz Blanke, Friedrich Kur. Munich, Verlag Rogner \& Bernhard, 1972 et Darmstadt, Wissenschaftliche Buchgesellschaft, 1973. Cette édition en 2 volumes comporte, sous la forme d'un volume séparé, une annexe Anhang und Dokumente (pp. $145^{*}$ à $397^{*}$ ) qui fait suite à l'Introduction du vol. 1 (pp. $7^{*}$ à $\left.144^{*}\right)$.

STÖRIG, Hans Joachim (éd. 1969). Das Problem des Übersetzens (Wege der Forschung 8). Darmstadt, Wissenschaftliche Buchgesellschaft. 